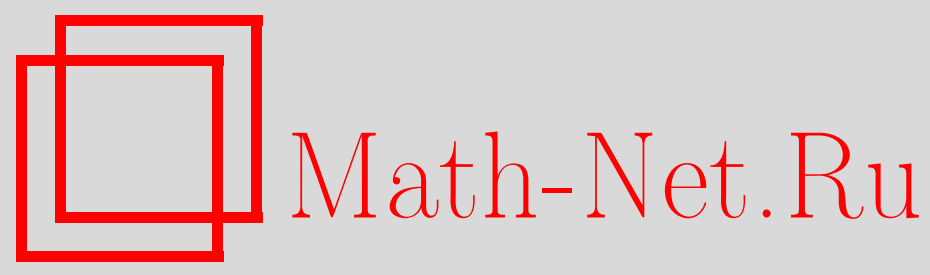

Ю. А. Альпин, Х. Д. Икрамов, Об унитарном подобии матричных семейств, Матем. заметки, 2003, том 74, выпуск $6,815-826$

DOI: https://doi.org/10.4213/mzm310

Использование Общероссийского математического портала Math-Net.Ru подразумевает, что вы прочитали и согласны с пользовательским соглашением http://www . mathnet.ru/rus/agreement

Параметры загрузки:

IP : 54.196 .121 .252

26 апреля 2023 г., $16: 14: 38$

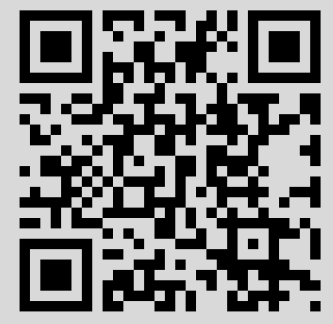


УДК 512.64

\title{
ОБ УНИТАРНОМ ПОДОБИИ МАТРИЧНЫХ СЕМЕЙСТВ
}

\author{
Ю.А. Альпин, Х. Д. Икрамов
}

Классический критерий Шпехта для унитарного подобия двух комплексных $(n \times n)$-матриц обобщается на случай унитарного подобия между двумя матричными ансамблями длины $m$, обладающими свойством нормальности. Это свойство состоит в том, что алгебра, порождаемая ансамблем, замкнута относительно матричной операции сопряжения. Подобно известному резултату Пирси, дополняющему теорему Шпехта, показано, что предлагаемое обобщение может быть превращено в конечньй критерий. При этом количество вычислений зависит не только от $n$, но и длины $l$ проверяемых алгебр. В случае пары матриц оно может быть значительно меньше трудоемкости критерия Шпехта-Пирси.

Библиография: 13 названий.

Два семейства комплексных матриц порядка $n$

$$
\left\{A_{1}, \ldots, A_{m}\right\} \quad \text { и } \quad\left\{B_{1}, \ldots, B_{m}\right\}
$$

назьваются унитарно подобными, если существует такая унитарная матрища $U$, что

$$
U^{*} A_{i} U=B_{i}, \quad i=1, \ldots, m .
$$

Если отказаться от требования унитарности преобразующей матрищы $U$ и заменить $U^{*}$ на $U^{-1}$, то получим определение (обычного) подобия матричных семейств.

Исследование условий унитарного подобия и поиск унитарных инвариантов имеют уже довольно давнюю историю (см. обзор [1]). Отметим здесь лишь работу [2] как пример геометрического подхода к проблеме. Мы же в этой работе продолжаем линию исследования, идущую от теоремы Шпехта (см. ниже теорему 2), и используем элементы теории представлений полугрупп и алгебр. Основные результаты содержатся в теоремах 4 и 5 и относятся к нормальньм семействам матриц. Это понятие обобщает понятие нормальной матрицы.

1. Унитарное подобие и обычное подобие семейств матриц. Теорема Шпехта. Как видно из следующего предложения, вопрос об унитарном подобии двух матриц сводится к вопросу о подобии двух пар матрищ.

ПРЕДЛОЖЕНИЕ 1. Для любых двух $(n \times n)$-матрии, $A$ и $B$ следующие утверждения әквивалентны:

1) $A$ и В унитарно подобнь;

2) семейства $\left\{A, A^{*}\right\}$ u $\left\{B, B^{*}\right\}$ унитарно подобны;

3) семейства $\left\{A, A^{*}\right\} u\left\{B, B^{*}\right\}$ подобны. 
ДокАЗАТЕЛЬСТво. Импликации 1$) \Rightarrow 2$ ) и 2) $\Rightarrow 3$ ) очевидны. Покажем, что 3) влечет 1). Пусть для невырожденной матрицы $P$ имеем

$$
P^{-1} A P=B, \quad P^{-1} A^{*} P=B^{*} .
$$

Тогда

$$
U^{*} A U=B,
$$

где $U$ - унитарный сомножитель в полярном разложении $P=S U$. Действительно, из равенств (1) следует, что

$$
P P^{*} A=A P P^{*} \text {. }
$$

Поскольку эрмитов сомножитель $S$, будучи квадратным корнем из $P P^{*}$, может быть представлен в виде многочлена от $P P^{*}$, то из последнего равенства следует, что $S A=$ $A S$. Отсюда вьводим желаемое:

$$
B=P^{-1} A P=U^{*} S^{-1} A S U=U^{*} A U
$$

В качестве следствия получаем: если нормальные матрицы $A$ и $B$ подобны, то они и унитарно подобны. В самом деле, нормальность матрицы $A$ равносильна, как известно, существованию такого многочлена $g(x)$, зависящего лишь от спектра $A$, что $g(A)=A^{*}$. Если $A$ и $B$ подобны, то их спектры совпадают и мы имеем равенство $g(B)=B^{*}$. Но тог да

$$
P^{-1} A P=B \quad \Rightarrow \quad P^{-1} A^{*} P=P^{-1} g(A) P=g(B)=B^{*},
$$

т.е. вьполнены равенства (1) и следствие (2) из этих равенств. Доказанное автоматически переносится на семейства матриц следующим образом.

ПРЕДЛОЖЕНИЕ 2. Если два семейства нормальных матриц, подобны, то они унитарно подобны.

Опишем один способ сведения проблемы унитарного подобия двух матрищ к проблеме подобия двух пар матриц. Пусть дано эрмитово разложение $A=H_{1}+i H_{2}$, где матрицы

$$
H_{1}=\frac{1}{2}\left(A+A^{*}\right), \quad H_{2}=\frac{1}{2 i}\left(A-A^{*}\right)
$$

- так назьваемые эрмитовы компоненты матрицы $A$.

ПРЕДЛОЖЕНИЕ 3. Для любыХ матрии $A$ и В следующие утвержддения әквивалентны:

1) $A$ и В унитарно подобны;

2) пары $\left\{H_{1}, H_{2}\right\}$ u $\left\{G_{1}, G_{2}\right\}$ әрмитовых компонент матрии, $A$ и $B$ унитарно подобны;

3) пары әрмитовых компонент матрии, $A$ и $B$ подобны. 
ДокАЗАтЕльСтво. Справедливость импликации 3) $\Rightarrow 2$ ) вытекает из предложения 2, поскольку эрмитовы матрицы нормальны. То, что 2) влечет 1) и 1) влечет 3 ), следует из определения эрмитовых компонент.

Наиболее известньм критерием унитарного подобия двух комплексных матриц является, по-видимому, теорема Шпехта. Чтобы обсудить эту теорему, введем обозначения, удобные и для дальнейшего изложения.

Мы будем задавать (конечное) семейство матриц как отображение $\alpha: X \rightarrow M_{n}(\mathbb{C})$, где $X$ - конечное множество, $M_{n}(\mathbb{C})$ - множество комплексньх матриц порядка $n$. Элементы $X$ называются буквами или некоммутирующими переменными. Семейство $\alpha$ можно также записать в виде $\alpha=\{\alpha(x), x \in X\}$.

Обозначим символом $\langle X\rangle$ свободньй моноид слов, порожденный множеством $X$. Роль единищы в $\langle X\rangle$ играет пустое слово $e$. Далее, пусть $\mathbb{C}[X]$ - свободная ассоциативная алгебра, порожденная множеством $X$, другими словами, алгебра формальных полиномов от некоммутирующих переменных из $X$. Слова в этой алгебре понимаются как мономы с коэффициентом 1.

Отображение $\alpha: X \rightarrow M_{n}$ единственным образом продолжается до гомоморфизма моноида $\langle X\rangle$ в мультипликативньй моноид $M_{n}(\mathbb{C})$ и далее до гомоморфизма алгебры $\mathbb{C}[X]$ в алгебру $M_{n}(\mathbb{C})$. Иначе говоря, матричное представление множества $X$ продолжается до матричного представления моноида $\langle X\rangle$ и матричного представления алгебры $\mathbb{C}[X]$. Не опасаясь путаницы, последние два представления можно обозначать той же буквой $\alpha$.

Предположим, что семейства $\alpha: X \rightarrow M_{n}$ и $\beta: X \rightarrow M_{n}$ подобны, т.е. существует $P \in M_{n}(\mathbb{C})$ такая, что $P^{-1} \alpha(x) P=\beta(x)$ для всех $x \in X$.

Тогда, очевидно, и для произвольного полинома $f \in \mathbb{C}[X]$ верно равенство

$$
P^{-1} \alpha(f) P=\beta(f) .
$$

В частности, разумеется, $f$ может быть словом из $\langle X\rangle$. Таким образом, оказьвается, что вопрос о подобии матричных семейств (т.е. матричных представлений множества $X$ ) равносилен вопросу о подобии определяемых этими семействами матричных представлений моноида $\langle X\rangle$ и алгебры $\mathbb{C}[X]$. Вообще говоря, этот факт не упрощает исходную задачу. Однако в важном частном случае вполне приводимых семейств критерий подобия может быть выведен из известной теоремы теории представлений. Напомним, что семейство $\alpha$ назьвается приводимым, если существует нетривиальное $\alpha$-инвариантное подпространство, т.е. подпространство, инвариантное относительно матриц $\alpha(x), x \in X$, рассматриваемых как линейные операторы в $\mathbb{C}^{n}$. Семейство $\alpha$ назьвается вполне приводимым, если для всякого $\alpha$-инвариантного подпространства существует прямое дополнение, тоже $\alpha$-инвариантное.

ТЕорема 1. Вполне приводимые семейства $\alpha$ и $\beta$ подобны тогда и только тог$\partial a, \kappa о г \partial a$

$$
\operatorname{tr} \alpha(w)=\operatorname{tr} \beta(w) \quad \forall w \in\langle X\rangle
$$

ДоКАЗАТЕЛЬСТВо получается приложением к представлениям моноида $\langle X\rangle$ известной теоремы [3]: если следы матрищ, соответствующих одному и тому же произвольно взятому элементу полугруппы, совпадают для двух вполне приводимых представлений полугруппы, то эти представления подобны. 
Теорема 2 (ШПеХт [4], [5]). Комплексные матрииы $А$ и В унитарно подобны тогда и только тогда, когда семейства

$$
\left\{\alpha\left(x_{1}\right)=A, \alpha\left(x_{2}\right)=A^{*}\right\} \quad u \quad\left\{\beta\left(x_{1}\right)=B, \beta\left(x_{2}\right)=B^{*}\right\}
$$

удовлетворяют условию

$$
\operatorname{tr} \alpha(w)=\operatorname{tr} \beta(w) \quad \forall w \in\left\langle\left\{x_{1}, x_{2}\right\}\right\rangle .
$$

Ввиду теоремы 1 и предложения 1 теорема Шпехта представляется совершенно прозрачной: вьполнение условий (5) влечет подобие вполне приводимых семейств (4), но это подобие равносильно унитарному подобию матрищ $A$ и $B$.

Если же мы соединим теорему 1 и предложение 3 , то получим другой критерий “шшехтовского типа" с вполне аналогичньм доказательством.

Теорема 2'. Комплексные матрицы $A$ и В унитарно подобны тогда и только тогда, когда семейства әрмитовых компонент этих матрии,

$$
\left\{\alpha\left(x_{1}\right)=H_{1}, \alpha\left(x_{2}\right)=H_{2}\right\} \quad u \quad\left\{\beta\left(x_{1}\right)=G_{1}, \beta\left(x_{2}\right)=G_{2}\right\}
$$

удовлетворяют условию (5).

2. Теорема об унитарном подобии нормальных матричных семейств. $\mathrm{B}$ предыдущем пункте мы имели дело с такими парами $\left\{A_{1}, A_{2}\right\}$, что матрицы $A_{1}$ и $A_{2}$ взаимно сопряжены или обе являются нормальными матрищами. Введем более общий класс семейств. Скажем, что семейство $\alpha: X \rightarrow M_{n}(\mathbb{C})$ нормальное, если

$$
\forall x \in X \quad \exists f \in \mathbb{C}[X] \quad(\alpha(x))^{*}=\alpha(f)
$$

Другими словами, нормальное семейство таково, что матрицы, сопряженные с матрицами семейства, могут быть выражены как полиномы от матриц семейства. Еще иначе: $\alpha$ - нормальное семейство, если подалгебра

$$
A=\{\alpha(f), f \in \mathbb{C}[X]\} \subseteq M_{n}(\mathbb{C})
$$

порожденная матрицами семейства, замкнута относительно операции сопряжения.

Выясним отношения между свойствами нормальности и полной приводимости матричных семейств.

Суммой семейств (представлений) $\alpha_{i}: X \rightarrow M_{n_{i}}(\mathbb{C}), i=1, \ldots, s$, называется представление $\alpha=\alpha_{1}+\cdots+\alpha_{s}$, определяемое формулой

$$
\alpha(x)=\alpha_{1}(x) \oplus \cdots \oplus \alpha_{s}(x), \quad x \in X
$$

где символ $\oplus$ обозначает прямое суммирование матриц.

Известна следующая характеристика вполне приводимых семейств [6]. 
ПРЕДЛОЖЕНИЕ 4. Семейство $\alpha: X \rightarrow M_{n}(\mathbb{C})$ вполне приводимо тогда и только тогда, когда оно подобно сумме неприводимых семейств.

Если $\alpha$ - неприводимое семейство, то согласно теореме Бернсайда [3], [6] оно порождает всю алгебру матриц, т.е. в этом случае $A=M_{n}(\mathbb{C})$.

Следовательно, всякое неприводимое семейство нормально. Всякое же нормальное семейство вполне приводимо. Более того, в предположении, что на $\mathbb{C}^{n}$ задано стандартное скалярное произведение $(a, b)$, верно следующее утверждение. Если $\alpha$-нормальное семейство, то ортогональное дополнение любого $\alpha$-инвариантного подпространства тоже $\alpha$-инвариантно. Действительно, пусть $L$ - инвариантное подпространство, $a \in L$, $b \in L^{\perp}$ и $f-$ такой полином из $\mathbb{C}[X]$, что $\alpha^{*}(x)=\alpha(f)^{1}$. Тогда

$$
(a, \alpha(x) b)=\left(\alpha^{*}(x) a, b\right)=(\alpha(f) a, b)=0,
$$

поскольку $\alpha(f) a \in L$. Это доказьвает, ввиду произвольности $x \in X$, что $L^{\perp}$ есть $\alpha$-инвариантное подпространство. Из приведенных рассуждений с очевидностью вытекает

ПРЕДЛОЖЕНИЕ 5. Если $\alpha: X \rightarrow M_{n}(\mathbb{C})$ - нормальное семейство, то пространство $\mathbb{C}^{n}$ разлагается в ортогональную сумму $\alpha$-инвариантных подпространств, каждое из которых уже не содержит нетривиальных $\alpha$-инвариантных подпространств.

В переводе на матричньй язык предложение 5 утверждает, что произвольное нормальное семейство унитарно подобно сумме неприводимых семейств. Однако описания нормальных семейств по типу предложения 4 не получается, поскольку сумма неприводимых семейств не обязана быть нормальным семейством. Для того, чтобы получить желаемое описание, докажем вначале

ПРЕДЛОЖЕНИЕ 6. Подобные нормальные семейства $\alpha: X \rightarrow M_{n}(\mathbb{C}) u \beta: X \rightarrow$ $M_{n}(\mathbb{C})$ тогда и только тогда унитарно подобны, когда семейство $\alpha+\beta$ нормальное.

ДокАЗАТЕЛЬСТво. Пусть $U$ - унитарная матрица, преобразующая $\alpha$ в $\beta$, и $f$-такой многочлен, что $\alpha^{*}(x)=\alpha(f)$. Тогда

$$
\beta^{*}(x)=U^{*} \alpha^{*}(x) U=U^{*} \alpha(f) U=\beta(f) .
$$

Следовательно,

$$
(\alpha+\beta)^{*}(x)=(\alpha+\beta)(f),
$$

что и доказьвает ввиду произвольного выбора $x \in X$ нормальность семейства $\alpha+\beta$.

Теперь предположим, что $\alpha+\beta$ - нормальное семейство: для всякого $x \in X$ существует $f \in \mathbb{C}[X]$ со свойством (6). Поскольку

$$
(\alpha+\beta)(f)=\alpha(f) \oplus \beta(f), \quad(\alpha+\beta)^{*}(x)=\alpha^{*}(x) \oplus \beta^{*}(x),
$$

то из (6) следует

$$
\alpha^{*}(x)=\alpha(f), \quad \beta^{*}(x)=\beta(f) .
$$

\footnotetext{
${ }^{1}$ Для краткости мы пишем $\alpha^{*}(x)$ вместо $(\alpha(x))^{*}$.
} 
Пусть матрища $P$ преобразует $\alpha$ в $\beta$ :

$$
P^{-1} \alpha(x) P=\beta(x), \quad x \in X .
$$

С учетом равенств (7) имеем

$$
P^{-1} \alpha^{*}(x) P=P^{-1} \alpha(f) P=\beta(f)=\beta^{*}(x) .
$$

Таким образом, для матриц $\alpha(x), \beta(x)$ выполнены равенства $(1)$, и завершая рассуждение так же, как доказательство предложения 1 , получаем, что в качестве матрищы, преобразуюшей $\alpha$ в $\beta$, можно взять унитарньй сомножитель полярного разложения матрицы $P$.

Сформулируем очевидное, но полезное

ПРЕДЛОЖЕНИЕ 7. Если $\alpha_{1}+\cdots+\alpha_{s}-$ нормальное семейство, то и любая частичная сумма $\alpha_{i_{1}}+\cdots+\alpha_{i_{t}}, 1 \leqslant i_{1}<\cdots<i_{t} \leqslant s$, является нормальным семейством.

Следующее утверждение - вариант теоремы Фробениуса-Шура [3]. Применительно к матричньм алгебрам она изложена в книге [7].

Tеорема 3. Пусть $\alpha_{i}: X \rightarrow M_{n_{i}}(\mathbb{C}), i=1, \ldots, l$, - неприводимые попарно неподобные семейства. Тогда для произвольных матрии, $C_{1}, \ldots, C_{l}$ порядков $n_{1}, \ldots, n_{l}$ найдется такой полином $h \in \mathbb{C}[X]$, что

$$
\left(\alpha_{1}+\cdots+\alpha_{l}\right)(h)=C_{1} \oplus \cdots \oplus C_{l} .
$$

Семейство $\alpha_{1}+\cdots+\alpha_{l}$ из теоремы 3 является нормальным, поскольку для всякого $x$ найдется такой полином $h$, что

$$
\alpha^{*}(x)=\alpha_{1}^{*}(x) \oplus \cdots \oplus \alpha_{l}^{*}(x)=\alpha_{1}(h) \oplus \cdots \oplus \alpha_{l}(h)=\alpha(h) .
$$

Из этого замечания и предложений 6,7 вытекает, что есть лишь одно препятствие для нормальности суммы неприводимых семейств - наличие в этой сумме подобных, но не унитарно подобных слагаемых. Итак, имеет место следующая характеристика нормальных семейств.

ПРЕДЛОЖЕНИЕ 8. Семейство а нормально тогда и только тогда, когда оно унитарно подобно такой сумме неприводимых семейств, в которой подобные слагаемые, если таковые имеются, унитарно подобны.

В отличие от частных случаев из предложений 1 и 2 , подобие нормальных семейств, вообще говоря, не равносильно их унитарному подобию. Рассмотрим, например, неприводимые семейства

$$
\left\{\alpha\left(x_{1}\right), \alpha\left(x_{2}\right)\right\} \quad \text { и } \quad\left\{\beta\left(x_{1}\right)=P^{-1} \alpha\left(x_{1}\right) P, \beta\left(x_{2}\right)=P^{-1} \alpha\left(x_{2}\right) P\right\},
$$

где матрица $P$ не пропорциональна унитарной матрице, т.е. $P P^{*}-$ не скалярная матрица. Согласно лемме Шура (см., например, [6]) любая матрища, преобразующая первую из наших пар во вторую, пропорциональна $P$. Следовательно, подобные нормальные семейства (8) не могут быть унитарно подобными.

Нашей целью является доказательство следующего критерия унитарного подобия нормальных семейств. 
Теорема 4. Нормальные семейства $\alpha: X \rightarrow M_{n}(\mathbb{C})$ и $\beta: X \rightarrow M_{n}(\mathbb{C})$ унитарно подобнь тогда и только тогда, когда

$$
\operatorname{tr} \alpha^{*}(v) \alpha(w)=\operatorname{tr} \beta^{*}(v) \beta(w) \quad \forall v, w \in\langle X\rangle .
$$

Прежде чем перейти к доказательству теоремы, заметим, что когда равенства (9) выполнены, то в силу линейности функции следа они останутся верными, если вместо слов $v, w$ подставить какие угодно полиномы $f, g \in \mathbb{C}[X]$. Функция

$$
(f, g)=\operatorname{tr} \alpha^{*}(g) \alpha(f)
$$

определяет скалярное произведение на комплексном линейном пространстве $\mathbb{C}[X]$. Стало быть, теорема 4 утверждает: семейства матриц $\alpha$ и $\beta$ унитарно подобны, если они определяют на $\mathbb{C}[X]$ одно и то же скалярное произведение.

ДОКАЗАТЕЛЬСТВО ТЕОРЕМЫ 4. НеобХодимость условий (9) для унитарного подобия проверяется без труда. Докажем достаточность. Условия (9), в частности, при $v=e$ содержат условия (3). Ввиду теоремы 1 и отмеченной вьше полной приводимости нормальных семейств эти условия обеспечивают подобие семейств $\alpha$ и $\beta$.

Чтобы доказать унитарноеподобие семейств $\alpha$ и $\beta$, рассмотрим вначале случай, когда $\alpha$ - неприводимое семейство. По теореме Бернсайда найдутся слова $w_{1}, \ldots, w_{n^{2}}$ такие, что матрицы

$$
\alpha\left(w_{1}\right), \quad \ldots, \quad \alpha\left(w_{n^{2}}\right)
$$

линейно независимы. Обозначим символом $\alpha^{\prime}\left(w_{i}\right)$ столбец высоты $n^{2}$, первые $n$ элементов которого составляют первый столбец $\alpha\left(w_{i}\right)$, следующие $n$ элементов - второй столбец, и т.д. Составим матрищу порядка $n^{2}$

$$
G=\left[\alpha^{\prime}\left(w_{1}\right), \ldots, \alpha^{\prime}\left(w_{n^{2}}\right)\right] .
$$

Матрица $G$, очевидно, невырожденная. Непосредственньми вычислениями проверяется равенство

$$
G^{*} n \alpha(w) G=\left\|\operatorname{tr} \alpha^{*}\left(w_{i}\right) \alpha(w) \alpha\left(w_{j}\right)\right\|
$$

для произвольного слова $w \in\langle X\rangle$. Здесь $n \alpha(w)$ - прямая сумма $n$ экземпляров $\alpha(w)$.

Пусть

$$
H=\left[\beta^{\prime}\left(w_{1}\right), \ldots, \beta^{\prime}\left(w_{n^{2}}\right)\right] .
$$

Из условий (9) вытекает для произвольного слова $w \in\langle X\rangle$ равенство

$$
G^{*} n \alpha(w) G=H^{*} n \beta(w) H .
$$

В частности, при $w=e$ вьполнено $G^{*} G=H^{*} H$. Из последнего равенства заключаем, что матрица $H$ невырожденная, а матрица $U=G H^{-1}$ унитарная. Но тогда из (10) имеем

$$
U^{*} n \alpha(w) U=n \beta(w) .
$$

Итак, семейства $n \alpha$ и $n \beta$ унитарно подобны и нормальны по построению. По предложению 6 семейство $n \alpha+n \beta$ тоже нормальное. Но тогда и семейство $\alpha+\beta$ нормальное - см. предложение 7. Как уже говорилось, равенство (9) обеспечивает подобие $\alpha$ и $\beta$. 
Снова применяя предложение 6 , теперь к семействам $\alpha$ и $\beta$, получаем, что $\alpha$ унитарно подобно $\beta$.

Пусть теперь $\alpha$ и $\beta$ - произвольные нормальные матричные семейства. Согласно предложению 8 каждое из них унитарным подобием может быть преобразовано в сумму неприводимых семейств, любые два из которых либо неподобны, либо унитарно подобны. Поэтому достаточно привести доказательство для семейств

$$
\alpha=\alpha_{1}+\cdots+\alpha_{s} \quad \text { и } \quad \beta=\beta_{1}+\cdots+\beta_{t},
$$

являющихся именно такими суммами.

Как упоминалось, из равенства (9) следует подобие семейств $\alpha$ и $\beta$. Из доказательства соответствующей теоремы о роли следа вполне приводимого представления [3] видно, что об этом подобии можно сказать точнее: в выражениях (11) необходимо $s=t$, и существует такая перестановка $\sigma$ на множестве $\{1, \ldots, s\}$, что $\alpha_{i}$ подобно $\beta_{\sigma(i)}$. Ради удобства обозначений будем предполагать, что $\alpha_{1}, \ldots, \alpha_{l}, l \leqslant s,-$ полный список попарно неподобных слагаемых. Этого можно добиться перестановкой диагональных блоков, т.е. преобразованием посредством перестановочной (стало быть, унитарной) матрищы. Следовательно, это предположение не уменьшает общности рассмотрений.

Далее, пусть $m_{i}, 1 \leqslant i \leqslant l,-$ число слагаемых в $\alpha$, подобных $\alpha_{i}$. Тогда равенства (9) можно переписать в виде

$$
\sum_{i=1}^{l} m_{i} \operatorname{tr} \alpha_{i}^{*}(v) \alpha_{i}(w)=\sum_{i=1}^{l} m_{i} \operatorname{tr} \beta_{\sigma(i)}^{*}(v) \beta_{\sigma(i)}(w) \quad \forall v, w \in\langle X\rangle .
$$

Заметим, что по причине линейности функции следа равенства (12) останутся верными, если вместо слов $v$ и $w$ подставить какие угодно полиномы.

Фиксируем произвольным образом $\alpha_{k}, 1 \leqslant k \leqslant l$, и слово $w \in\langle X\rangle$. Согласно теореме 3 найдется такой полином $h \in \mathbb{C}[X]$, что

$$
\begin{gathered}
\alpha_{k}(h)=\alpha_{k}(w), \\
\alpha_{i}(h)=0 \quad \text { для всех } i \neq k .
\end{gathered}
$$

Пусть матрица $P_{k}$ преобразует семейство $\alpha_{k}$ в семейство $\beta_{\sigma(k)}$. В частности,

$$
P_{k}^{-1} \alpha_{k}(h) P_{k}=\beta_{\sigma(k)}(h), \quad P_{k}^{-1} \alpha_{k}(w) P_{k}=\beta_{\sigma(k)}(w) .
$$

В силу (13) отсюда получаем

$$
\beta_{\sigma(k)}(h)=\beta_{\sigma(k)}(w),
$$

а учитывая (14), имеем

$$
\beta_{\sigma(i)}(h)=P_{i}^{-1} \alpha_{i}(h) P_{i}=0 \quad \text { для всех } i \neq k .
$$

Подставляя в (12) вместо $w$ полином $h$, а затем, учитывая равенства (13)-(16) и сокращая обе части на множитель $m_{k}$, приходим к равенству

$$
\operatorname{tr} \alpha_{k}^{*}(v) \alpha_{k}(w)=\operatorname{tr} \beta_{\sigma(k)}^{*}(v) \beta_{\sigma(k)}(w)
$$


где, напомним, на слова $v$ и $w$ не наложено никаких условий. Но в таком случае согласно первой части доказательства неприводимые семейства $\alpha_{k}$ и $\beta_{\sigma(k)}$ унитарно подобны. Обозначим $U_{k}$ унитарную матрицу, преобразующую $\alpha_{k}$ в $\beta_{\sigma(k)}$, а буквой $F$ - такую перестановочную матрицу, что

$$
F^{*}\left(\beta_{1}(x) \oplus \cdots \oplus \beta_{s}(x)\right) F=\beta_{\sigma(1)}(x) \oplus \cdots \oplus \beta_{\sigma(s)}(x) \quad \forall x \in X .
$$

Тогда, как легко видеть, унитарная матрица

$$
\left(U_{1} \oplus \cdots \oplus U_{s}\right) F^{*}
$$

преобразует семейство $\alpha$ в семейство $\beta$. Это завершает доказательство.

3. Конечные критерии унитарного подобия. Теорема Шпехта была усовершенствована Пирси [8], [5], который превратил ее в конечньй критерий, доказав, что равенства (5) достаточно проверить для слов длины не больше $2 n^{2}$. Тем самым было доказано существование конечной полной системы унитарных инвариантов. Мы покажем, что и теорему 4 можно превратить в конечный критерий. Обозначим множество слов длины не больше $k$ символом $X^{k}$, а количество таких слов символом $\bar{k}$. Пусть

$$
d_{k}=\operatorname{dim} \operatorname{span}\left\{\alpha(p), p \in X^{k}\right\}
$$

Тогда, очевидно,

$$
1=d_{0} \leqslant d_{1} \leqslant \cdots .
$$

При этом, если

$$
1=d_{0}<d_{1}<\cdots<d_{l}=d_{l+1}
$$

Tо

$$
d_{l}=d_{l+1}=\cdots \quad \text { и } \quad \operatorname{dim} \mathscr{A}=d_{l} .
$$

Наименьшее число $l$, для которого $d_{l}=d_{l+1}$, будем назьвать длиной семейства $\alpha$.

Нам будет удобно предполагать, что множество $\langle X\rangle$ перенумеровано: $\langle X\rangle=\left\{p_{0}\right.$, $\left.p_{1}, \ldots\right\}$, причем так, что более короткое слово имеет меньший номер, чем более длинное. $\mathrm{B}$ частности, $p_{0}=e$. Составим матрицу Грама

$$
G_{k}=\left\|\operatorname{tr} \alpha^{*}\left(p_{i}\right) \alpha\left(p_{j}\right)\right\|
$$

системы “векторов" $\alpha\left(p_{0}\right), \alpha\left(p_{1}\right), \ldots, \alpha\left(p_{\bar{k}-1}\right)$. Ранг матрицы Грама системы векторов равен максимальному числу линейно независимых векторов этой системы, следовательHO,

$$
\operatorname{rank} G_{k}=d_{k}
$$

Из этого равенства вытекает

ПРЕДЛОЖЕНИЕ 9. Длина семейства $\alpha: X \rightarrow M_{n}(\mathbb{C})$ равна наименьшему числу $l$ со свойством

$$
\operatorname{rank} G_{l}=\operatorname{rank} G_{l+1}
$$

Теперь докажем 
ПРЕДЛОЖЕНИЕ 10. Функиионал $\operatorname{tr} \alpha^{*}(v) \alpha(w)$ определен для любъх $v, w \in\langle X\rangle$, если известны его значения на словах $v \in X^{l}, w \in X^{l+1}$, әде $l$ - длина семейства $\alpha$.

ДокАЗАТЕЛЬСТво. Представим матрицу $G_{k}$ в виде

$$
G_{k}=H_{k}^{*} H_{k}
$$

где

$$
H_{k}=\left[\alpha^{\prime}\left(p_{0}\right), \alpha^{\prime}\left(p_{1}\right), \ldots, \alpha^{\prime}\left(p_{\bar{k}-1}\right)\right] .
$$

Определим вспомогательное семейство матриц $\hat{\alpha}: X \rightarrow M_{\bar{l}}(\mathbb{C})$ из уравнений

$$
n \alpha(x) H_{l}=H_{l} \hat{\alpha}(x), \quad x \in X .
$$

Поскольку $n \alpha(x) H_{l}=\left[\alpha^{\prime}(x), \alpha^{\prime}\left(x p_{1}\right), \ldots \alpha^{\prime}\left(x p_{\bar{l}-1}\right)\right], x \in X$, и согласно свойствам $(17)$, (18) числа $l$ система уравнений (19) в самом деле разрешима. Умножая уравнения (19) на $H_{l}^{*}$, получим систему

$$
H_{l}^{*} n \alpha(x) H_{l}=G_{l} \hat{\alpha}(x), \quad x \in X .
$$

Системы уравнений (19) и (20) ввиду совместности первой из них равносильны (9). Поскольку

$$
H_{l}^{*} n \alpha(x) H_{l}=\left\|\operatorname{tr} \alpha^{*}\left(p_{i}\right) \alpha\left(x p_{j}\right)\right\|,
$$

то можно утверждать, что семейство $\hat{\alpha}$ вполне определяется числами

$$
\operatorname{tr} \alpha^{*}(v) \alpha(w), \quad v \in X^{l}, \quad w \in X^{l+1},
$$

где $l$ - длина $\alpha$.

Равенства (19), понятно, останутся верными, если в них $x$ заменить на любое слово $w$. Перемножая равенства

$$
n \alpha(w) H_{l}=H_{l} \hat{\alpha}(w) \quad \text { и } \quad H_{l}^{*} n \alpha^{*}(v)=\hat{\alpha}^{*}(v) H_{l}^{*},
$$

получаем

$$
H_{l}^{*} n \alpha^{*}(v) n \alpha(w) H_{l}=\hat{\alpha}^{*}(v) H_{l}^{*} H_{l} \hat{\alpha}(w)
$$

или

$$
\left\|\operatorname{tr} \alpha^{*}\left(v p_{i}\right) \alpha\left(w p_{j}\right)\right\|=\hat{\alpha}^{*}(v) G_{l} \hat{\alpha}(w) .
$$

Отметим, что элемент $(1,1)$ матрищы $(21)$ равен $\operatorname{tr} \alpha^{*}(v) \alpha(w)$.

Итак, зная матрицу $G_{l}$ и матричное семейство $\hat{\alpha}$, можно вычислить значение $\operatorname{tr} \alpha^{*}(v) \alpha(w)$ для любых $v, w \in\langle X\rangle$. Но $G_{l}$ и $\hat{\alpha}$ определяются, как показано вьше, значениями, указанными в формулировкепредложения 10. Это завершает доказательство.

Теперь нетрудно доказать конечный критерий унитарного подобия нормальных матричных семейств.

Теорема 5. Пусть $\alpha: X \rightarrow M_{n}(\mathbb{C})$ - нормальное семейство длины l. Оно унитарно подобно семейству $\beta: X \rightarrow M_{n}(\mathbb{C})$, если

$$
\operatorname{tr} \alpha^{*}(v) \alpha(w)=\operatorname{tr} \beta^{*}(v) \beta(w) \quad \forall v, w \in X^{l+1} .
$$


ДокАЗАтЕльство. Равенства (22) обеспечивают совпадение матриц Грама для семейств $\alpha$ и $\beta$ :

$$
G_{k}(\alpha)=G_{k}(\beta), \quad k=1, \ldots, l, l+1 .
$$

Следовательно, по предложению 9 длина семейства $\beta$ равна $l$. Применяя затем предложение 10 , заключаем, что равенства (22) вьполняются для всех $v, w \in\langle X\rangle$. Но в таком случае по теореме 4 семейства $\alpha$ и $\beta$ унитарно подобны.

Рассмотрим семейства типа (4). Сопоставим слову $v=x_{i_{1}} \ldots x_{i_{k}}$ слово $v^{\prime}=$ $\bar{x}_{i_{k}} \ldots \bar{x}_{i_{1}}$, где $\bar{x}_{i_{t}}=x_{2}$, если $x_{i_{t}}=x_{1}$, и $\bar{x}_{i_{t}}=x_{1}$, если $x_{i_{t}}=x_{2}$. Тогда $\alpha^{*}(v)=\alpha\left(v^{\prime}\right)$, $\beta^{*}(v)=\beta\left(v^{\prime}\right)$, и, следовательно, всякое равенство из (22) эквивалентно некоторому равенству $\operatorname{tr} \alpha\left(v^{\prime} w\right)=\operatorname{tr} \beta\left(v^{\prime} w\right)$. Таким образом, имеет место следующий конечный вариант теоремы Шпехта.

СлеДСТВИЕ. Для унитарного подобия матрии, $A$ и В достаточно потребовать выполнения равенств

$$
\operatorname{tr} \alpha(w)=\operatorname{tr} \beta(w) \quad \forall w \in X^{2 l+2},
$$

əде $l$ - длина семейства $\alpha$.

В теореме 5 и следствии из нее семейства $\alpha$ и $\beta$, разумеется, равноправны и можно положить, что $l$ - длина $\beta$.

ЗАмЕчАниЕ. В предварительной публикации [10] на тему данной статьи утверждалось, что равенства (22) достаточно проверить для $v \in X^{l}, w \in X^{l+1}$. В качестве следствия приводился результат из [11] (см. также [12]), состоящий в том, что равенства (23) достаточно проверить для $w \in X^{2 l+1}$. В ходе тщательной проверки наших рассуждений выяснилось, что в данньй момент мы располагаем доказательствами лишь приведенных вьше чуть более слабых утверждений.

Вычисление длины $l$ матричного семейства с целью проверки унитарного подобия может потребовать больших вычислений, поэтому интерес представляют верхние оценки для $l$. Естественный способ получения таких оценок основан на неравенстве

$$
l \leqslant \operatorname{dim} \mathscr{A}-1
$$

непосредственно следуюшем из соотношений $(17),(18)$. Очевидно, что

$$
l \leqslant n^{2}-1
$$

Нетривиальная и тоже априорная - зависящая лишш от порядка $n$ матриц - оценка длины матричного семейства получена недавно в [11]:

$$
l \leqslant n \sqrt{\frac{2 n^{2}}{n-1}+\frac{1}{4}}+\frac{n}{2}-2 .
$$

Применительно к проверке унитарного подобия двух матрищ неравенство (24) позволяет ограничиться словами длины не больше $2 n^{2}$ (это результат Пирси [8]), а неравенство (25) дает более точную оценку, по порядку равную $n^{3 / 2}[11],[12]$.

Лучшие оценки можно получить, если учитывать “индивидуальность” матричного семейства. Приведем два примера нормальных семейств, для которых оценка длины линейна. 
ПРЕДЛОЖЕНИЕ 11. Если $\alpha$ - семейство нормальных коммутирующих матрии, mo

$$
l \leqslant n-1
$$

ДокАЗАТЕЛьСтво. Действительно, в силу спектральной теоремы нормальные коммутируюшие матрицы приводятся к диагональному виду одним подобием. Следовательно, размерность порождаемой ими алгебры не превьшает $n$, а длина семейства не больше $n-1$.

ПРЕДЛОЖЕНИЕ 12. Если $\alpha\left(x_{1}\right)=A, \alpha\left(x_{2}\right)=A^{*}$, әде $A-$ матрица с квадратичным минимальным многочленом, то длина семейства а удовлетворяет условию

$$
l \leqslant \begin{cases}2(n-1), & \text { если } n \text { нечетное } \\ 2 n-1, & \text { если } п \text { четное. }\end{cases}
$$

Это утверждение - следствие результата из [13] о размерности алгебры, порождаемой парой матриц с квадратичными минимальньпи многочленами.

\section{СПИСОК ЦИТИРОВАННОЙ ЛИТЕРАТУРЫ}

[1] Shapiro H. A survey of canonical forms and invariants for unitary similarity // Linear Algebra and Appl. 1991. V. 147. P. 101-167.

[2] Калужнин Л.А., Хавиди Х. Геометрическая теория унитарной эквивалентности матриц // Докл. РАН. 1996. Т. 169. № 5. С. 1009-1012.

[3] Ван дер Варден Б. Л. Алгебра. М.: Наука, 1979.

[4] Specht W. Zur Theorie der Matrizen, II // Jahresber. Deutsch. Math. -Verein. 1940. V. 50. P. 19-23.

[5] Хорн Р., Джонсон Ч. Матричньй анализ. М.: Мир, 1989.

[6] Винберг Э. Б. Курс алгебры. М.: Факториал, 1999.

[7] Вейль Г. Классические группы, их инварианты и представления. М.: ИЛ, 1947.

[8] Pearcy C. A. A complete set of unitary invariants for operators generating finite $W^{*}$-algebras of type I // Pacific J. Math. 1962. V. 12. P. 1405-1416.

[9] Воеводин В.В., Кузнецов Ю. А. Матрицы и вычисления. М.: Наука, 1984.

[10] Альпин Ю. А., Икрамов Х. Д. Об унитарном подобии семейств матриц // Докл. РАН. 2002. T. 384. № 2. C. 151-152.

[11] Freedman A., Gupta R. N., Guralnik R. M. Shirshov's theorem and representations of semigroups // Pacific J. Math. 1997. V. 181. № 3. P. 159-176.

[12] Pappacena C. An upper bound for the length of a finite-dimensional algebra // J. Algebra. 1997. V. 197. P. 535-545.

[13] Gaines F.T., Laffey T.J., Shapiro H.M. Pairs of matrices with quadratic minimal polynomials // Linear Algebra and Appl. 1983. V. 52/53. P. 289-292.

Ю. А. Алпин: Казанский государственный университет 